\title{
Liste der im Handbuch angewandten Literatur-Abkürzungen.
}

\section{Zeitschriften.}

American Chemical Journal

American Journal of Science (Silliman) . . . . .

Annalen der Chemie (Liebig)

Annalen der Physik und Chemie (Poggendortt)

Ann$$
\text { " }
$$$$
"
$$

(Wiedemann)

Annales de Chimie et de Physique.

Beiblïtter zu den Annalen der Physik

Berichte der Deutschen Chemischen Gesellschaft

Berichte der Deutschen Physikal. Gesellschaft.

Bulletin de la Societé chimique de Paris (de France)

Chemical News, The

Chemiker Zeitung .

Chemische Industrie, Die

Chemisches Zentralblatt

Chemische Zeitschrift .

Comptes rendus de l'Academie des Sciences (Paris)

Dinglers Polytechnisches Journal

Elektrochemische Zeitschrift

Gazzetta chimica italiana

Jahresbericht über die Fortschritte der Chemie etc.

Journal de Chimie physique

Journal de physique

Juurnal für prabtische Chemie

Journal of Physical Chemistry

Journal of the American Chemical Society

Journal of the Chemical Society (London)

Journal of the Society of Chemical Industry

Mitteilungen aus dem Kgl. Materialprüfungsamt zu Groblichterfelde

Monatshefte für Chemie

Moniteur scientifique.

Osterreichische Chemiker Zeitung

Pharmaceutical Journal Transactions . . . . . . . . . . .

Pharmuzeutische Zentralhalle

\section{Abkürzungen}

Am. Ch. J.

Am. J. Science

Ann.

Pogg.

Wied.

Ann. Phys.

Ann. chim. phys.

Beibl.

Ber.

Ber. Phys. Ges.

Bull.

Chem. N.

Ch. Z.

Ch. Ind.

Zentr.

Ch. Ztschr.

C. $\mathrm{r}$.

Dingl.

Elektr. Ztschr.

Gazz.

Jahr.

J. Chim. phys.

J. phys.

J.pr. Ch.

J. Physic. Chem.

J. Am. Ch. S.

J. Chem. S.

J. Soc. Cb. Ind.

Mitt. Mater. Amt Mfte.

Monit.

Öst. Ch. Ztg.

Pharm. J. Trans.

Pharm. Z.

Pharm. Zentr. 
Ablürzungen

Philosophical Magazine . . . . . . . . . . . . . . . . Phil. Mag.

Physikalische Zeitschrift . . . . . . . . . . . . . . . . Phys. Ztschr.

Proceedings of the Chemical Society (London) . . . . . . . Proc. Chem. S.

Recueil des travaux chimiques des Pays-Bas. . . . . . . . Ree trav. chim.

Sitzungsberichte der Kgl. Akademie der Wissenschaften, Wien . . Sitzgb. Wien.

Sitzungsberichte der Kgl. PreuBischen Akademie der Wissenschaften,

Berlin . . . . . . . . . . . . . . . . . . . . . Sitzgb. Berlin

Sitzungsberichte der Kgl. Bayrischen Akademie der Wissenschaften,

mathemat.-naturwissenschaftliche Abteilung . . . . . . Sitzgb. Bayr.

Stahl und Eisen. . . . . . . . . . . . . . . . . . . St. E.

Zeitschrift für analytische Chemie . . . . . . . . . . . Z. anal. Ch.

Zeitschrift für angewandte Chemie . . . . . . . . . . . . Z. angew. Ch.

Zeitschrift für anorganische Chemie . . . . . . . . . . . . Z. anorg. Ch.

Zeitschrift für Chemie und Industrie der Kolloide . . . . . . Z. Kolloid.

Zeitschrift für chemische Apparatenkunde . . . . . . . . . Z. Appar.

Zeitschrift für physikalischen und chemischen Unterricht. . . . Z. Unterr.

Zeitschrift für Elektrochemie . . . . . . . . . . . . . . Z. Elektr.

Zeitschrift für Instrumentenkunde . . . . . . . . . . . . Z. Instr.

Zeitschrift für physikalische Chemie . . . . . . . . . . . Z. phys. Ch.

\section{Handbücher.}

Abderhalden, Handbuch der biochemischen Arbeitsmethoden

Abegr-Auerbach, Haudbuch der anorganischen Chemie

Arendt-Doermer, Technik der anorganischen Experimentalchemie. 1910 . . . . . . . . . .

Bender.Erdmann, Chemische Präparatenkunde. Zwei

Bände. 1893 . . . . . . . . . . . . . Bender-Erdmann

Berzelius, Iuehrbuch der Chemie. 4. Aufl. Zehn

Bände. 1835-1841 . . . . . . . . . . . Berzelius

Dammer, Handbuch der anorganischen Chemie . . Dammer

Fehling, Handwörterbuch der Chemie. . . . . . Fehling

Gmelin-Kraut, Handbuch der anorganischen Chemie.

6. Auflage . . . . . . . . . . . . . . Gmelin-Kraut

Gmeliu-Kraut, Handbuch der anorganischen Chemie.

7. Auflage, herausgegeben von Friedheim u. Peters

Graham-Otto, Ausführliches Lehrbuch der Chemie. 5. Auflage von Michaelis . . . . . . . .

Landolt-Börnstein, Physik.-chem. Tabellen. 3. Aufl. 1905 . . . . . . . . . . . . . . . .

desgl. 4. Autl. 1912 . . . . . . . . . . . . Landolt-Börnstein-Roth

Moissan, Traité de chimie minérale. 1906 . . . . Moissan

Muspratt, Handbuch der technischen Chemie. 1891 . Muspratt

Ostwald-Luther, Physikochemische Messungen. 3. Aufl. 1910 . . . . . . . . . . . . Ostwald-Luther

Roscoë-Schorlemmer, Ausführliches Lehrbuch der

Chemie . . . . . . . . . . . . . . . Roscö̈-Schorlemmer

Travers-Estreicher, Experimentelle Untersuchung von

Gasen. 1905. . . . . . . . . . . Travers-Estreicher 